\title{
Multi-photon nanosurgery in live brain
}

\author{
Anna Letizia Allegra Mascaro', Leonardo Sacconi ${ }^{1,2}$ and Francesco S. Pavone ${ }^{1,2 *}$ \\ 1 European Laboratory for Non-linear Spectroscopy, University of Florence, Florence, Italy \\ 2 Department of Physics, University of Florence, Florence, Italy
}

\author{
Edited by: \\ David Boas, Massachusetts General \\ Hospital, USA; Massachusetts Institute \\ of Technology, USA; Harvard Medical \\ School, USA \\ Reviewed by: \\ Chris Shaffer, Cornell University, USA \\ Willy Supatto, National Centre for \\ Scientific Research, France \\ *Correspondence: \\ Francesco S. Pavone, Department of \\ Physics and European Laboratory for \\ Non-linear Spectroscopy, University of \\ Florence, Via Sansone 1 - 50019 Sesto \\ Fno., Florence, Italy. \\ e-mail: francesco.pavone@unifi.it
}

In the last few years two-photon microscopy has been used to perform in vivo high spatial resolution imaging of neurons, glial cells and vascular structures in the intact neocortex. Recently, in parallel to its applications in imaging, multi-photon absorption has been used as a tool for the selective disruption of neural processes and blood vessels in living animals. In this review we present some basic features of multi-photon nanosurgery and we illustrate the advantages offered by this novel methodology in neuroscience research. We show how the spatial localization of multi-photon excitation can be exploited to perform selective lesions on cortical neurons in living mice expressing fluorescent proteins. This methodology is applied to disrupt a single neuron without causing any visible collateral damage to the surrounding structures. The spatial precision of this method allows to dissect single processes as well as individual dendritic spines, preserving the structural integrity of the main neuronal arbor. The same approach can be used to breach the blood-brain barrier through a targeted photo-disruption of blood vessels walls. We show how the vascular system can be perturbed through laser ablation leading toward two different models of stroke: intravascular clot and extravasation. Following the temporal evolution of the injured system (either a neuron or a blood vessel) through time lapse in vivo imaging, the physiological response of the target structure and the rearrangement of the surrounding area can be characterized. Multi-photon nanosurgery in live brain represents a useful tool to produce different models of neurodegenerative disease.

Keywords: laser surgery, laser ablation, laser dissection, in vivo imaging, two-photon microscopy

\section{INTRODUCTION}

The link between optics and biology has always been extremely strong. In fact, it was through the use of the first optical microscopes in the 17th century that fundamental concepts such as the existence of the cell itself were defined. In the 1960s, the advent of the laser has totally revolutionized biology and medicine. Recently, femtosecond lasers have gained ground due to their versatile application to a large variety of problems. The first application developed for short-pulsed lasers in biology was multi-photon fluorescence microscopy (Denk et al., 1990). The non-linear nature of multiphoton processes provides an absorption volume spatially confined to the focal region (Zipfel et al., 2003). The localization of the excitation is maintained even in strongly scattering tissues, allowing deep, high-resolution in vivo microscopy (Helmchen and Denk, 2005; Svoboda and Yasuda, 2006).

In parallel to its application in imaging, multi-photon absorption has also been used as a tool to manipulate the biological sample under investigation; in particular, this technique has been applied to the selective disruption of labeled cells and intracellular structures. The first published report of femtosecond laser subcellular nanosurgery was by König et al. (1999), who demonstrated the potential of this technique by ablating nanometer-sized regions of the genome within the nucleus of living cells. Following this seminal work, laser nanosurgery has been applied in living cells to investigate the biological function of subcellular compartments, like mitochondria or microtubules (Tolić-Nørrelykke et al., 2004; Watanabe and Arakawa, 2004; Sacconi et al., 2005; Shen et al., 2005; Shimada et al., 2005; Kumar et al., 2006).
A similar approach has also been applied in living animals, where two-photon imaging and laser-induced lesions have been combined. For example, femtosecond laser ablations have been applied for studying the complex events associated with embryo development in vivo (Supatto et al., 2005; Kohli and Elezzabi, 2008). Other groups have taken advantage of multi-photon absorption to ablate or dissect individual neurons in worms to study axon regeneration (Yanik et al., 2004) and define the role of specific neurons in behavior. As an example, the function of a single neuron can be identified by observing behavioral changes after the surgery, as shown in the study by Chung et al. (2006), on the thermo-tactic behavior of nematodes.

Recently, the potential of this technique has been applied to the mammalian central nervous system (CNS). Thanks to this approach the investigation of many outstanding problems in neurobiology and human neurodegenerative disease appears now more accessible. For example, Sacconi et al., (2007) demonstrated a method for performing laser nanosurgery in the CNS of anesthetized mice. In this work the spatial localization of multi-photon excitation was exploited to perform selective lesions on the processes of cortical neurons in living mice expressing fluorescent proteins. Furthermore, laser-induced lesions have been used to damage the blood-brain barrier, to investigate the role of microglia (Davalos et al., 2005; Nimmerjahn et al., 2005) and also to produce targeted photo-disruptions as a model of stroke (Nishimura et al., 2006). Although acutely silent, small strokes are neurologically important as cortical microinfarcts and microhemorrhages may increase the risk of developing dementia 
(Cullen et al., 2005; Gold et al., 2007). Non-linear laser ablation provides a method to induce vascular lesions one vessel at a time and to study cerebral microvascular diseases. Two-photon excited fluorescence microscopy, coupled to micro-vessel occlusion technique, enables to study the impact of microvascular lesions at cellular level, in vivo.

In this review we present some basic features of multi-photon nanosurgery and show some examples that illustrate the advantages offered by this novel methodology. In the first part we will review the experimental system used to perform multi-photon nanosurgery, giving detailed information about our custom-made multi-photon nanodissector and defining a method to gain optical access to the brain. In the second part we will show the results of performing imaging and nanosurgery in neural networks and in the vascular system. While illustrating some applications of femtosecond lasers to disrupt cellular structures of the CNS in vivo, we will provide a description of the morphological consequences of the injury event. In the end we will discuss the physical mechanisms of neural nanosurgery to help explaining the consequences of femtosecond laser ablation.

The opportunity provided by multi-photon nanosurgery for interacting with neural networks offers great potential to the investigation of brain remodeling. The nanosurgery technique applied to the nervous system in vivo can reveal detailed insight of the dynamic rearrangements of the mammalian CNS. In vivo time lapse imaging of cerebral fine structures in the days that follow a point disruption may represent an optimal tool to characterize the network response to injury and mimic a neurodegenerative pathology.

\section{EXPERIMENTAL METHODS OPTICAL WINDOW PREPARATION}

The optical penetration of two-photon microscopy in live brain is limited by the opacity of the intact skull of adult mice. One way to obtain optical access to the neural and vascular network requires a craniotomy surgery; this method, called "open skull” technique (see Figure 1), implies the permanent removal of a circular portion of skull (leaving the dura mater unperturbed) and the replacement of this isle of bone with a cover glass (Lendvai et al., 2000; Trachtenberg et al., 2002; Holtmaat et al., 2006).

Alternatively, a small bone area $\left(0.1-0.3 \mathrm{~mm}^{2}\right)$ can be thinned down to a $20 \mu \mathrm{m}$ layer ("thinned skull" technique, see Grutzendler et al., 2002; Zuo et al., 2005). While the thinned skull preparation requires bone thinning at every experimental session, the permanent cranial window allows repeated imaging at arbitrary time intervals. Furthermore the open skull provides a larger field of view $\left(0.8-12 \mathrm{~mm}^{2}\right)$ as well as an excellent optical access for weeks to months.

The experimental protocol of the open skull technique, recently illustrated by Holtmaat et al. (2009), will be described briefly. GFP-M line mice aged 3-5 months are deeply anesthetized by intraperitoneal injection of ketamine $(90 \mathrm{mg} / \mathrm{kg})$ and xylazine ( $9 \mathrm{mg} / \mathrm{kg})$. A small dose of dexamethasone $(0.04 \mathrm{ml}$ at $2 \mathrm{mg} / \mathrm{ml})$ is administered prior to surgery to minimize brain swelling. The mouse head is fixed in a stereotactic frame. The eyes are protected from dehydration through application of eye drops. After cutting out a strip of skin, the periosteum is removed by scraping the

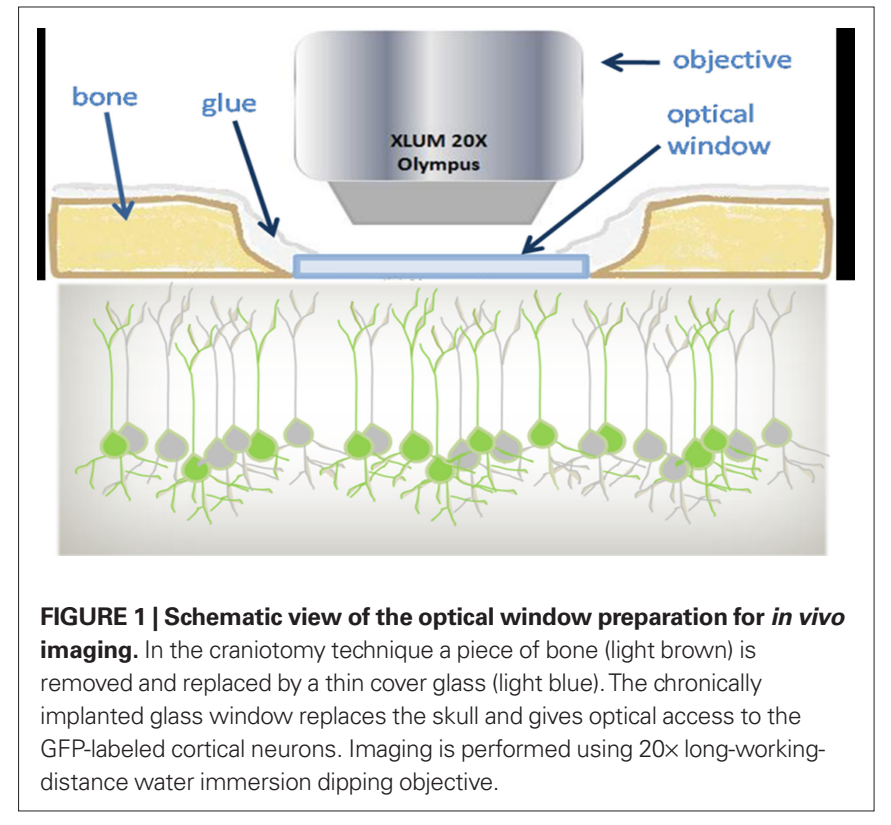

skull with a scalpel blade. Then, a circular portion of the skull is thinned out with a dental drill. The skull island is lifted up and removed, carefully avoiding to touch the meninges with the tweezers. The optical chamber is constructed by laying a cover glass upon the intact dura, as shown in Figure 1. A mixture of dental acrylic and cyanoacrylate glue is used to seal the cranial window. A metal bar is fixed above the skull to stabilize the animal for subsequent imaging sessions. To minimize the inflammatory phenomena that may take place after the surgery, the mice are treated with an anti-inflammatory drug, Carprofen $(5 \mathrm{mg} / \mathrm{kg})$, administered daily through subcutaneous injection. The apical dendrites of pyramidal neurons are imaged every 1-2 days to detect and study the remodeling events of the neural network up to more than 1 month after the surgery. Recently, a useful video showing this methodology has been posted on the Journal of Visualized Experiment (Mostany and Portera-Cailliau, 2008).

While the neuronal network is partially labeled in the GFP-M mice, the injection of a vital dye is required to image and manipulate the vascular structures. To this end, blood plasma was labeled through tail vein injection of a $0.2 \mathrm{ml}$ bolus of $5 \%(\mathrm{w} / \mathrm{v})$ Texas Red dextran (70 kDa) (D-1830, Invitrogen) in physiological saline (Kleinfeld et al., 1998; Zhang et al., 2005; Nishimura et al., 2006; Schaffer et al., 2006). This high molecular weight dye provides stable labeling (typically $3 \mathrm{~h}$ ).

\section{OPTICAL NANOSURGERY SETUP}

In this section we describe a typical experimental setup that combines multi-photon imaging and nanosurgery. This particular experimental system was used in our laboratory to perform in vivo multi-photon nanosurgery on cortical neurons (Sacconi et al., 2007). The setup, shown in Figure 2, is a custom-made upright two-photon microscope.

A mode-locked Ti:Sapphire laser (Chameleon, Coherent Inc) provides the excitation light (120 fs width pulses at $90 \mathrm{MHz}$ repetition rate). A $\lambda / 4$ waveplate coupled to a rotatable polarizer finely 


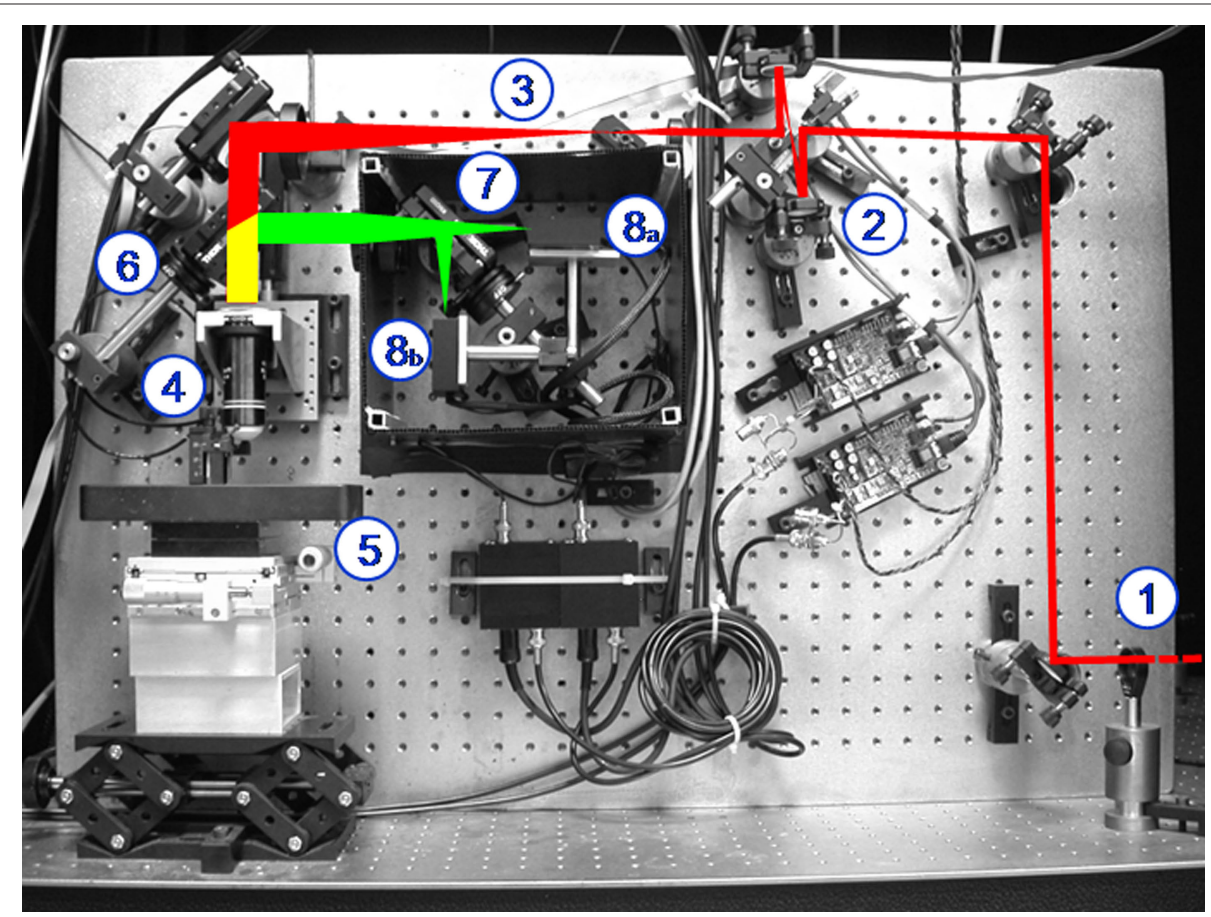

FIGURE 2 | Custom-made two photon apparatus. The figure shows the laser beam [in red, coming from the bottom right side of the panel (1)] that is first scanned by two galvanometric mirrors (2), then expanded by a telescope (3), and finally focused by the objective (4) onto the specimen (5). The emitted light (yellow) is separated from the exciting beam by a first dichroic mirror (6) and then split by a second dichroic mirror (7) in the red and green components. Two photomultipliers detect the split fluorescence emissions $(8 a, b)$. moved by a stepper motor provides a tunable beam intensity control. The laser beam is scanned in the xy-plane by a non-resonant scanning head. The scanning system consists of two closed-loop feedback galvanometer mirrors (VM500, GSI Lumonics), rotated about orthogonal pivots and coupled by a pair of spherical mirrors. The laser beam is then expanded by a telescope composed by a scanning lens and a microscope tube lens. Finally an objective lens (XLUM 20X, NA 0.95, WD $2 \mathrm{~mm}$, Olympus) focuses the beam onto the specimen. The anesthetized mouse is positioned in the stereotactic holder screwing a metal bar placed on the mouse head (see above); in this way the same cerebral region of interest can be found in every imaging session through fixed coordinates with high precision. The stereotactic holder is fixed on a stage through a magnetic platform laying upon a lab jack that allows rough axial positioning of the optical window under the objective. Fine xy-displacement is accomplished through two independent micromanipulators on the magnetic stage. A closed-loop piezoelectric stage (P-721, Physik Instrumente) allows axial displacements of the objective up to $400 \mu \mathrm{m}$ with nanometric precision. Fluorescence light is separated from the laser optical path by a first dichroic beam splitter (685DCXRU, Chroma Technology) positioned as close as possible to the objective lens (non-de-scanning mode). A two-photon florescence cut-off filter (E700SP-2P, Chroma Technology) eliminates reflected laser light. A second dichroic mirror (FF562-Di02-25 $\times 36$, Semrock) is used to split the two spectral components of the fluorescence signal, the red (570-685 nm) and the green (400-560 nm) emission light. The fluorescence signals are finally collected by two photomultiplier modules (H7710-13,
Hamamatsu Photonics). All the microscope optics are fixed onto a custom vertical honeycomb steel breadboard. The electronic components of the setup are computer-controlled during microscopy and nanosurgery experiments. Custom-made software has been developed in LabVIEW 7.1 (National Instruments).

The key requisites for the experimental setup to perform laser nanosurgery are the maximum stability and reproducibility $(\approx 0.1 \mu \mathrm{m})$ of beam positioning in the $x, y$ and $z$ axes. This condition is fulfilled by closed-loop feedback systems on the piezoelectric stage and on the galvo mirrors. On the other hand, motorized $\mathrm{z}$ positioning systems and resonant galvo mirrors, sometimes present in commercial systems, do not fit the requirements for nanosurgery. However, commercial multi-photon setups provided with high stability positioning system can be used to perform both imaging and nanosurgery in the low-density plasma regime, as previously shown (Supatto et al., 2008). Other technical requirements involve, for example, laser pulse dispersion and maximum power deliverable on the sample. Due to the large variety of commercially available systems these parameters should be evaluated on a case-by-case basis.

\section{OPTICAL NANOSURGERY PROCEDURE}

The nanosurgery experiment starts with an in vivo imaging session of the exposed mouse brain under the optical window. Z-stacks are acquired to obtain 3D-reconstructions of the labeled sample. During the imaging session, the excitation wavelength $(\lambda)$ is chosen to maximize the multi-photon cross-section of the labeled structures (in our experiment $\lambda=935 \mathrm{~nm}$ ). After selecting 
the $x y z$ coordinates for the desired lesion site, laser dissection is performed by irradiating the selected point with a high energy dose of Ti:Sapphire laser at the same wavelength used for imaging. In a typical experiment, the laser power is increased 5-10 times more than the power used for imaging and the shutter is opened for a period of the order of hundreds of millisecond. The increase of energy dose delivered on the sample in the dissection with respect to imaging arises largely from an increase in exposure time, with a lower contribution from the increase in laser power itself. In fact, the pixel dwell time is $\approx 5 \mu$ s during imaging, whereas it is increased to hundreds of millisecond to perform the nanosurgery. As a consequence, the number of pulses (and the associated energy dose) delivered per unit area is drastically increased (five orders of magnitude). After performing the nanosurgery, the laser power is decreased back and a $3 \mathrm{D}$-image of the sample is acquired to visualize the effects on the irradiated structure.

It is worth mentioning that the experimental parameters for the nanosurgery (laser power and exposure time) critically depend on the target. For example, the depth, the fluorescence level and the dimension of the target structure remarkably affect the energy dose threshold to perform the nanosurgery. Furthermore, the anesthetic levels and the optical window preparation critically affect the stability of the sample and, thus, the outcome of the irradiation. Practically, the laser power is set close to the maximum available value ( $\approx 300 \mathrm{~mW}$, measured after the objective lens) and an exposure time of $100 \mathrm{~ms}$ is tested to induce ablation. Then an imaging session is performed to observe the effects of the laser irradiation. If no morphological changes were induced, the laser surgery is repeated with an increased exposure time. This procedure is performed iteratively, until a detectable structural modification is observed. In a typical experiment the exposure time ranges from 100 to $1000 \mathrm{~ms}$.

\section{NANOSURGERY IN LIVE BRAIN NEURAL ABLATION}

The optical nanosurgery setup, in combination with the optical window preparation, has been used to perform in vivo imaging and ablation of fine neural structures in the cortex of live animals. The spatial localization of multi-photon excitation is exploited to perform in vivo selective lesions on small subsets of neurons in the somatosensory cortex of mice genetically expressing fluorescent proteins (GFP-M) without causing any visible collateral damage to the surrounding neuronal structures (Sacconi et al., 2007). Neurons are irradiated with a focused, controlled femtosecond energy dose and then the morphological consequences are characterized with time lapse $3 \mathrm{D}$ two-photon imaging. The potential of this method is such that different compartments of the CNS can be perturbed by either ablating a neural cell body (see Figure 3A) or disrupting the distal portion of a dendrite (see Figure $3 \mathrm{~B}$ ), or even providing the selectivity for the ablation of a single spine (see Figure 3C).

The possibility of dissecting selected structures depends, to a first approximation, on the depth of the structure and on its fluorescence emission intensity. Although two-photon microscopy allows imaging of layer-5 pyramidal neurons (600-800 $\mu \mathrm{m}$ below the pia), the nanosurgery is more limited in depth because of the loss of energy density along the beam path across the brain. Interneuronal somas placed in the layer-2/3 (200-450 $\mu \mathrm{m}$ below the pia) can be easily disrupted by laser nanosurgery. Figure 3A shows the time course of the ablation of a GFP-labeled interneuronal cell body. The interneuron was irradiated in proximity of the red lightning symbol just after the acquisition of the first image. The merge of the neuron before (red) and after (green) injury (right panel in Figure 3A) shows the disappearance of the cellular body after laser mediated ablation, while the surrounding network is negligibly affected. Laser nanosurgery can be also applied to apical dendrites of a layer- 5 pyramidal neuron. Figure 3B shows the time course of a point disruption of a single pyramidal dendrite. The effect of the ablation is the selective degeneration of the distal portion of the neurite, indicating that this may be an active process and not a passive deterioration (see Discussion in the next paragraph). The right panel in Figure 3B highlights the integrity of the remaining structure after dissection. Furthermore, the continued expression of fluorescent proteins indicates that the neuron is alive. Finally, the high spatial precision of this method was demonstrated by ablating individual dendritic spines, while sparing the adjacent spines and the structural integrity of the dendrite. Figure 3C shows a portion of a GFP-labeled pyramidal dendrite where several spines are present. The right panel of Figure 3C highlights the disappearance of a single spine after laser-induced lesion and emphasizes both the stability of the surrounding dendritic spines (indicated by yellow arrowheads) and the absence of any swelling in the dendrite.

This micro-scale model of neural degeneration allows to characterize the remodeling properties of the network after injury. In comparison with other models of neural ablation, e.g., massive neural depletion through 3-acetyl pyridine treatment (Hicks, 1955; Desclina and Escubia, 1974; Balaban, 1985), nanosurgery is highly specific and can be finely tuned to a single neuron, leaving the surrounding structures unaltered.

\section{BLOOD VESSELS DISRUPTION}

Another important application of multi-photon microscopy is imaging of blood flow through cortical blood vessels labeled with a vital dye. The nanosurgery technique applied to the vascular system leads to subsurface blood vessel disruption in living animals (Nishimura et al., 2006). This model of stroke allows to reproduce different ischemic conditions and, at the same time, to investigate with day-by-day detail the reactive capabilities of the system. Femtosecond laser pulses are focused on a blood vessel to achieve two different forms of vascular insult; the irradiation may lead either to vessel rupture, which provides a model of hemorrhage (see Figure 4A), or to formation of a blood clot (Figure 4B).

Two-photon real time imaging of blood flow through the surrounding vessels before and after the laser-induced vascular lesion permits to characterize the dynamics of the degenerative event. In the first example the complete rupture of a blood vessel leads to the release of blood plasma and red blood cells in the surrounding tissue, while flow is left intact in nearby vessels. The formation of a clot, on the other hand, determines a complete blockage of the flow in the targeted blood vessel. Recent studies show that a 
A
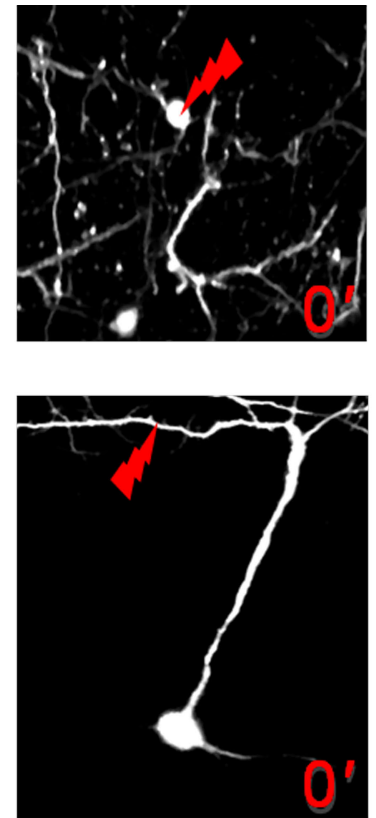

C

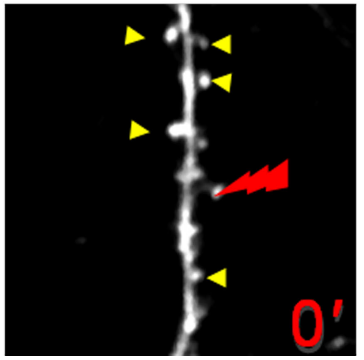

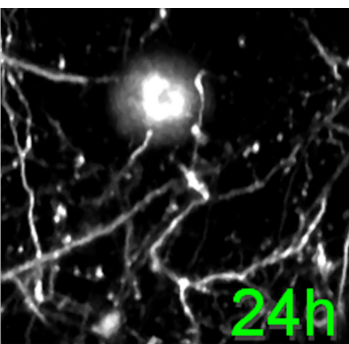
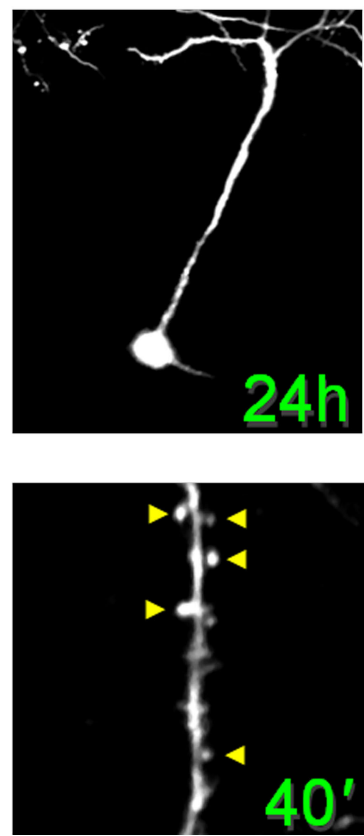
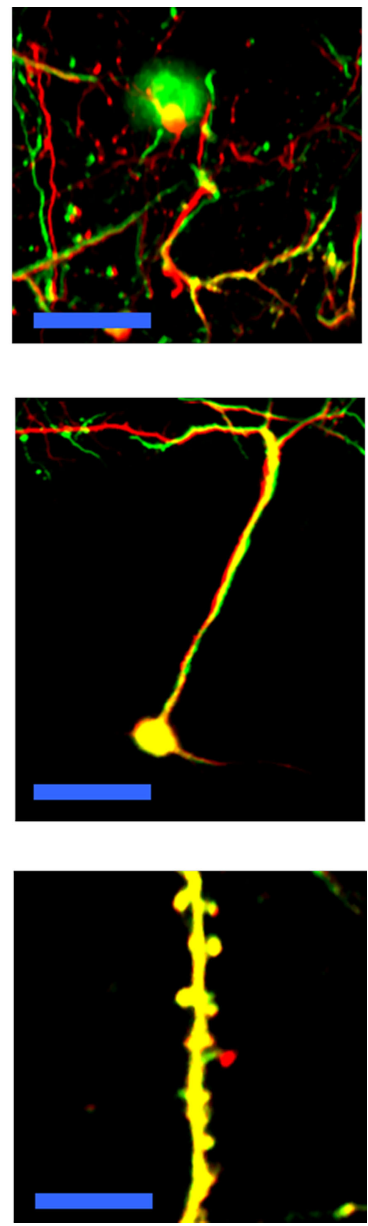

FIGURE 3 | Neural nanosurgery in GFP-M transgenic mice. (A) Laser dissection of an interneuron cell body. Each image is a maximum-intensity projection of a set of 20 optical sections acquired at $2 \mu \mathrm{m}$ z-step (at $150 \mu \mathrm{m}$ depth). The tip of the red lightning symbol represents the laser irradiation point. The first panel was acquired just before the laser irradiation. The middle panel was acquired $24 \mathrm{~h}$ after the laser irradiation. The last panel shows an overlay of the neuron before (red) and after (green) laser irradiation. Scale bar, $25 \mu \mathrm{m}$. (B) Laser-induced lesion of a single dendrite. Maximum-intensity z projection (from 100 to $500 \mu \mathrm{m}$ ) of a pyramidal neuron before and $24 \mathrm{~h}$ after dendritic dissection. Scale bar, $60 \mu \mathrm{m}$. (C) Laser ablation of a single dendritic spine. Images of a portion of a dendrite before and $40 \mathrm{~min}$ after spine ablation. Scale bar, $15 \mu \mathrm{m}$. (B) and (C) are modified with permission from Sacconi et al. (2007). single micro-vessel lesion determines the disruption of flow at the nearby downstream vessels, but the presence of loops within the network may partially compensate for the lost vascular afference (Nishimura et al., 2006). The response of the neural network to this local blood deprivation is characterized through the days that follow the vascular insult. Time-lapse observation of the adjacent labeled neurons reveals a relatively high stability of the network in case of a confined damage, while loss of dendritic spines and degeneration of dendrites can be observed if the injury is more widespread (Zhang and Murphy, 2007).

While conventional methods allowed to create mainly largescale occlusions that destroyed extended areas of brain vasculature (Chen et al., 1986; Ping et al., 2008), the nanosurgery technique can finely tune the injury toward a single blood microvessel (Nishimura et al., 2006; Kleinfeld et al., 2008). The possibility of monitoring blood flow before and after an occlusion discloses a great potential for this technique, that may be applicable to the investigation of interventional therapies for micro-strokes.

\section{MECHANISM OF MULTI-PHOTON NANOSURGERY}

In order to explore the mechanism of multi-photon nanosurgery, we analyzed the morphological rearrangements of an apical pyramidal dendrite after the irradiation with a high energy dose of femtosecond pulsed laser. Based on the biological response, the morphological consequences of irradiating a single dendrite can be grouped into two categories: transient swelling with recovery, and complete dendritic dissection. Figure 5 shows the time course of the laserinduced degeneration of an apical dendrite in GFP-M transgenic somatosensory cortex. In the first case (see Figure 5A) we observe a transient swelling of the dendrite extending several micrometers from the point of irradiation along the dendrite in both directions. The irradiated dendrite temporarily modifies its shape but recovers its original features after a period of hours. While in this example the nanosurgery does not lead to dendritic dissection, in a second class of response the dendrite undergoes a degenerative process. As shown in Figure 5B, after laser irradiation, the terminal end of the dendrite distal to the dissection point followed a sequence of 

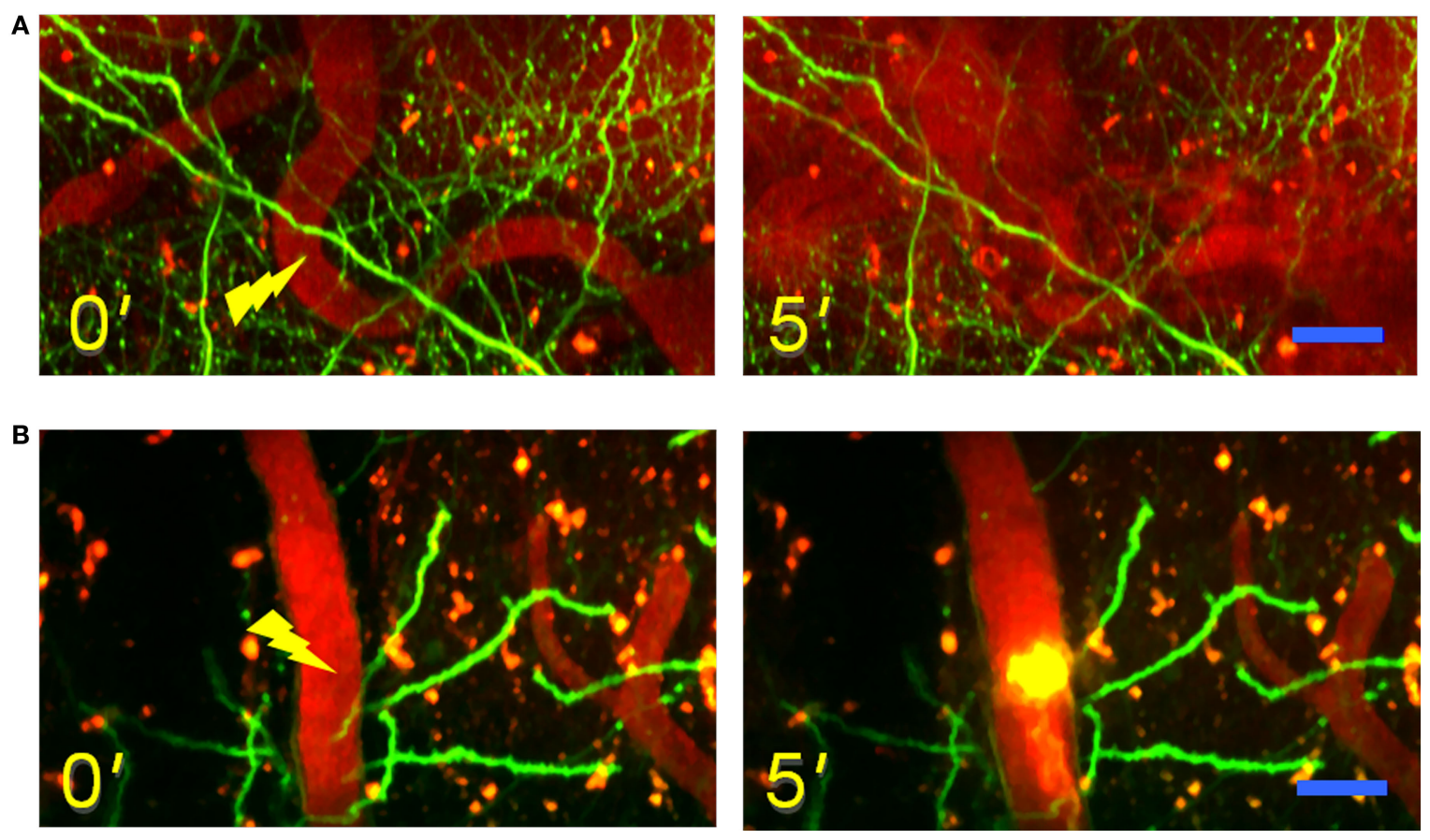

FIGURE 4 | Models of laser-induced vascular lesion. (A) Time lapse images of maximum intensity z-projections (from 20 to $60 \mu \mathrm{m}$ ) before (left) and after (right) the laser-induced ischemic hemorrhage. The figures show in green the GFP-labeled neuron in a GFP-M mouse and in red the vascular network labeled with Texas-red dextran dye. The tip of the yellow lightning symbol represents the laser irradiation point. The first image was acquired just before the laser irradiation. Scale bar, $20 \mu \mathrm{m}$. (B) Time lapse images of maximum intensity z-projections (from 20 to $100 \mu \mathrm{m}$ ) before (left) and after (right) the laser induction of an intravascular clot. Scale bar, $20 \mu \mathrm{m}$. swelling, degeneration, and disappearance. The energy dose necessary to produce these outcomes (swelling with recovery or neural ablation) cannot be established a priori. In fact, the same energy dose can determine the two different biological responses.

These irradiation outcomes may be interpreted on the basis of the physical mechanism underlying the nanosurgery technique. For simplicity, two regimes can be established for nanosurgery (Vogel and Venugopalan, 2003; Vogel et al., 2005). The low-density plasma regime uses long series of pulses from femtosecond oscillators with repetition rates of the order of $80 \mathrm{MHz}$ and pulse energies well below the optical breakdown threshold (König et al., 1999, 2001; Oehring et al., 2000; Smith et al., 2001; Tirlapur and König, 2002; Zeira et al., 2003; Sacconi et al., 2005, 2007; Supatto et al., 2005). The low repetition rate regime, instead, uses amplified series of pulses at repetition rates of kilohertz and pulse energies slightly above the optical breakdown threshold (Watanabe and Arakawa, 2004; Yanik et al., 2004; Heisterkamp et al., 2005; Shen et al., 2005). The mechanisms on which these two regimes rely are quite different. The low repetition rate regime effects are related to thermoelastically-induced formation of transient cavities. An extensive review focused on this regime has been recently published by Tsai et al. (2009). On the other hand, in our work we present several examples in which the dissection is performed in the low-density plasma regime. In this case the dissection is mediated by free-electron-induced chemical decomposition. The chemical decomposition can be induced through two different mechanisms: (1) the fragmentation of the biomolecule derives from its resonant interaction with quasi-free electrons: the capture of a quasi-free electron in an antibonding molecular orbital causes the rupture of a chemical bond and determines the degradation of the molecule; (2) the fragmentation of the biomolecule derives from its interaction with reactive oxygen species (ROS, i.e., free radicals that contain the oxygen atom), originated from laser-induced ionization and dissociation of water molecules.

Following this description, the outcome of the nanosurgery on apical dendrites can be interpreted by an alteration of membrane permeability due to chemical decomposition of the plasma membrane components. The laser-induced transient breach in the cell membrane leads to the dendritical swelling shown in Figure 5. Now, if the laser perturbation is small enough, the neuron may re-establish the physiological osmotic equilibrium and recover after a transient swelling, as shown in Figure 5A. Otherwise, the membrane disruption may lead to the formation of bead-like structures, followed by degeneration of the distal portion of the dendrite (see Figure 5B). This process, called Wallerian degeneration (Waller, 1850), occurs after axonal injury at the distal stump of the site of injury and usually begins within $24 \mathrm{~h}$ of a lesion. As recently reported in Kerschensteiner et al. (2005), axonal injuries initially lead to acute axonal degeneration, which is the rapid separation of the proximal and distal ends within $30 \mathrm{~min}$ after an injury. Degeneration follows with swelling of the axolemma, and eventually leads to bead-like formations. After axon degradation, granular disintegration of the axonal cytoskeleton and inner organelles occurs. This anterograde deterioration, elicited by the 

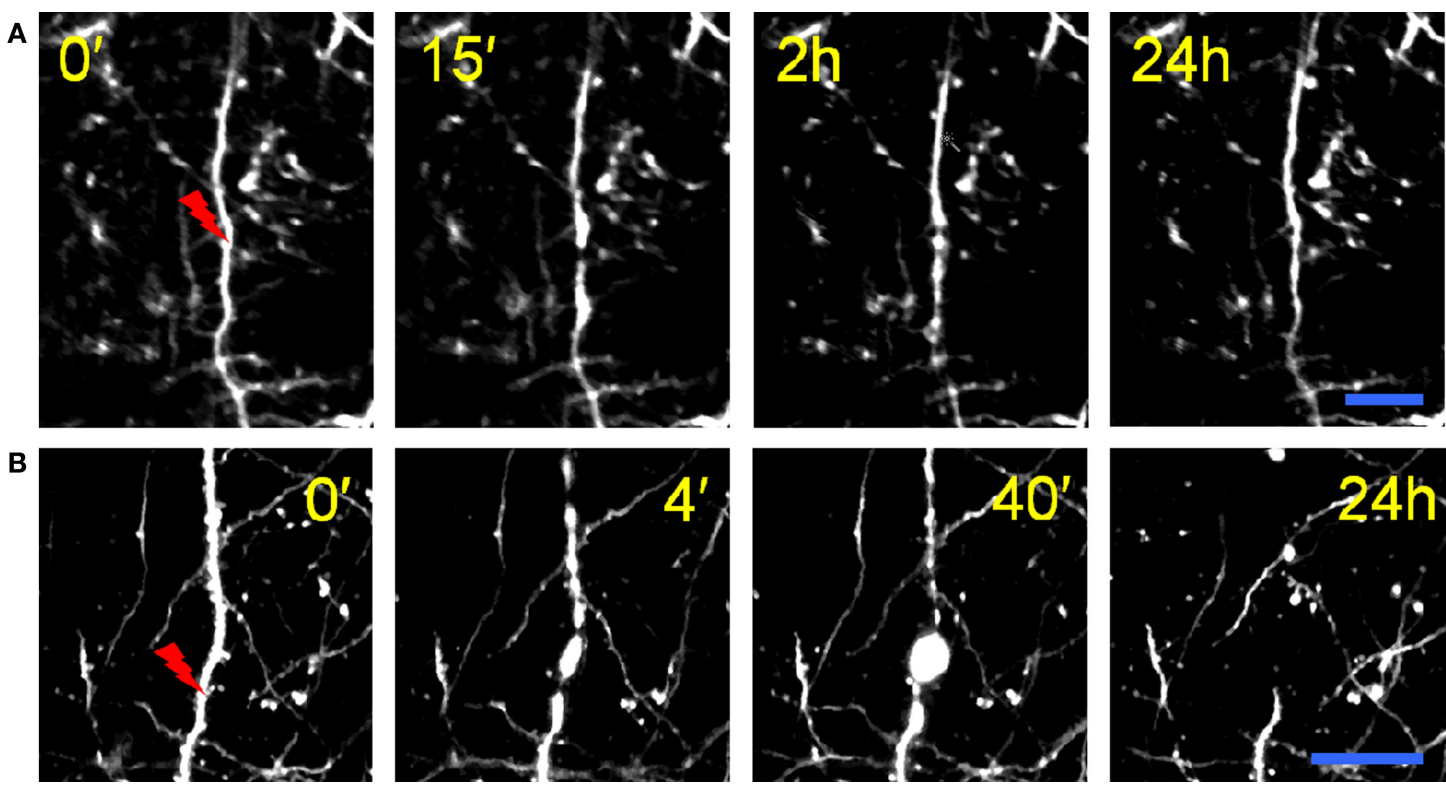

FIGURE 5 | Effects of the nanosurgery on apical dendrites. (A) Laser-induced dendritic swelling. Time-lapse of maximum intensity z-projections (from 140 to $160 \mu \mathrm{m}$ ) of irradiated dendrite in GFP-M transgenic mouse. The tip of the red lightning symbol represents the laser irradiation point. The first panel was acquired just before the laser irradiation. Scale bar, $10 \mu \mathrm{m}$. (B) Laser-induced lesion of a single dendrite. Time-lapse of maximum intensity z-projections (from 90 to $110 \mu \mathrm{m}$ ) of irradiated dendrite. Scale bar, $25 \mu \mathrm{m}$. (B) is modified from Sacconi et al. (2007) calcium influx (George and Griffin, 1994), is dependent on ubiquitin and calpain proteases, suggesting that axonal degeneration is an active process rather than a passive lack of support from the cell body (Zimmerman and Schlaepfer, 1984).

\section{CONCLUSIONS}

In the last years laser absorption has been exploited to interfere with biological processes and, thus, to define the effective mechanism of specific events. Multi-photon nanosurgery, applied to the CNS of living mammals, represents the ideal platform to setup different models of cortical injury. In vivo two-photon fluorescence imaging coupled to laser-induced nanosurgery, is a promising tool to study the reparative properties of the adult CNS, as it gives an insight of the real dynamics of the neural network plastic properties, in

\section{REFERENCES}

Balaban, C. D. (1985). Central neurotoxic effects of intraperitoneally administered 3-acetylpyridine, harmaline and niacinamide in Sprague-Dawley and Long-Evans rats: a critical review of central 3-acetylpyridine neurotoxicity. Brain Res. 356, 21-42.

Chen, S. T., Hsu, C. Y., Hogan, E. L., Maricq, H., and Balentine, J.D. (1986). A model of focal ischemic stroke in the rat: reproducible extensive cortical infarction. Stroke 17, 738-743.

Chung, S. H., Clark, D. A., Gabel, C. V., Mazur, E., and Samuel, A. D. (2006). The role of the AFD neuron in $C$. elegans thermotaxis analyzed using femtosecond laser ablation. BMC Neurosci. 7, 30
Cullen, K. M., Kócsi, Z., and Stone, J. (2005). Pericapillary haem-rich deposits: evidence for microhaemorrhages in aging human cerebral cortex. J. Cereb. Blood Flow Metab. 25, 1656-1667.

Davalos, D., Grutzendler, J., Yang, G., Kim, J. V., Zuo, Y., Jung, S., Littman, D. R., Dustin, M. L., and Gan, W. B. (2005).ATP mediates rapid microglial response to local brain injury in vivo. Nat. Neurosci. 8, $752-758$.

Denk, W., Strickler, H. J., and Webb, W. W. (1990). Two-photon laser scanning fluorescence microscopy. Science 248, 73-76.

Desclina, J. C., and Escubia J. (1974). Effects of 3-acetylpyridine on the central nervous system of the rat, as

physiological as well as injured conditions. Through multi-photon nanosurgery, we can reproduce interesting models of neural degeneration and finely characterize the evolution of the neural network in vivo after those injury events.

\section{ACKNOWLEDGMENTS}

The authors thank Francesco Vanzi for useful discussion about the manuscript. This research project has been supported by the European Community's Sixth Framework Program (Marie Curie Transfer of Knowledge Fellowship MTKD-CT-2004-BICAL509761), by "Consorzio Nazionale Interuniversitario per le Scienze Fisiche della Materia" (CNISM), the PRIN Program 2007, by the "Ente Cassa di Risparmio di Firenze" (private foundation), and by ASI project MoMa.

demonstrated by silver methods. Brain Res. 77, 349-364.

George, R., and Griffin, J. W. (1994). Delayed macrophage responses and myelin clearance during Wallerian degeneration in the central nervous system: the dorsal radiculotomy model. Exp. Neurol. 129, 225-236.

Gold, G., Giannakopoulos, P., Herrmann, F. R., Bouras, C., and Kövari, E. (2007) Identification of Alzheimer and vascular lesion thresholds for mixed dementia. Brain 130, 2830.

Grutzendler, J., Kasthuri, N., and Gan, W. B. (2002). Long-term dendritic spine stability in the adult cortex. Nature 420, 812-816.

Heisterkamp, A., Maxwell, I. Z., Mazur, E., Underwood, J. M., Nickerson, J. A.,
Kumar, S., and Ingber, D. E. (2005). Pulse energy dependence of subcellular dissection by femtosecond laser pulses. Opt. Express 13, 3690-3696.

Helmchen, F., and Denk, W. (2005). Deep tissue two-photon microscopy. Nat. Methods 2, 932-940.

Hicks S. P. (1955). Pathologic effects of antimetabolites. I. Acute lesions in the hypothalamus, peripheral ganglia, and adrenal medulla caused by 3-acetyl pyridine and prevented by nicotinamide. Am. J. Pathol. 31,189-199.

Holtmaat, A., Bonhoeffer, T., Chow, D. K., Chuckowree, J., De Paola, V., Hofer, S. B., Hübener, M., Keck, T., Knott, G., Lee, W. C., Mostany, R., Mrsic-Flogel, T. D., Nedivi, E., 
Portera-Cailliau, C., Svoboda, K., Trachtenberg, J. T., and Wilbrecht, L. (2009). Long-term, high-resolution imaging in the mouse neocortex through a chronic cranial window. Nat. Protoc. 4, 1128-1144.

Holtmaat, A., Wilbrecht, L., Knott, G. W., Welker, E., and Svoboda, K. (2006). Experience dependent and cell-typespecific spine growth in the neocortex. Nature 441, 979-983.

Kerschensteiner, M., Schwab, M. E., Lichtman, J.W., and Misgeld, T. (2005). In vivo imaging of axonal degeneration and regeneration in the injured spinal cord. Nat. Med. 11, 572-577.

Kleinfeld, D., Friedman, B., Lyden, P. D., and Shih,A.Y. (2008). "Targeted occlusion to surface and deep vessels in neocortex via linear and nonlinear optical absorption," in Animal Models of acute Neurological Injuries, eds J.Chen, Z. Xu, X.-M.Xu, and J.Zhang (New York: The Humana Press Inc), pp. 169-185.

Kleinfeld, D., Mitra, P. P., Helmchen, F., and Denk, W. (1998). Fluctuations and stimulus-induced changes in blood flow observed in individual capillaries in layers 2 through 4 of rat neocortex. Proc. Natl. Acad. Sci. U.S.A. 95, 15741-15746.

Kohli, V., and Elezzabi, A. Y. (2008). Laser surgery of zebrafish (Danio rerio) embryos using femtosecond laser pulses: Optimal parameters for exogenous material delivery, and the laser's effect on short- and long-term development. BMC Biotechnol. 8, 7.

König, K., Riemann, I., and Fritzsche, W. (2001). Nanodissection of human chromosomes with near-infrared femtosecond laser pulses. Opt. Lett. 26, 819-821.

König, K., Riemann, I., Fischer, P., and Halbhuber, K. H. (1999). Intracellular nanosurgery with near infrared femtosecond laser pulses. Cell. Mol. Biol. 45, 195-201.

Kumar, S., Maxwell, I. Z., Heisterkamp, A., Polte, T. R., Lele, T., Salanga, M., Mazur, E., and Ingber, D. E. (2006). Viscoelastic retraction of single living stress fibers and its impact on cell shape, cytoskeletal organization, and extracellular matrix mechanics. Biophys. J. 90, 3762-3773.

Lendvai, B., Stern, E., Chen, B., and Svoboda, K. (2000). Experiencedependent plasticity of dendritic spines in the developing rat barrel cortex in vivo. Nature 404, 876-881.
Li, P., and Murphy, T. H. (2008). TwoPhoton Imaging during Prolonged Middle Cerebral Artery Occlusion in Mice Reveals Recovery of Dendritic Structure after Reperfusion. J. Neurosci. 28, 11970 -11979.

Mostany, R., and Portera-Cailliau, C. (2008). A craniotomy surgery procedure for chronic brain imaging. JoVE. (2008) Available at: http://www.jove. com/index/details.stp?id=680, doi: 10.3791/680.

Nimmerjahn, A., Kirchhoff, F., and Helmchen, F. (2005). Resting microglial cells are highly dynamic surveillants of brain parenchyma in vivo. Science 308, 1314-1318.

Nishimura, N., Schaffer, C. B., Friedman, B., Tsai, P. S., Lyden, P. D., and Kleinfeld, D. (2006). Targeted insult to subsurface cortical blood vessels using ultrashort laser pulses: three models of stroke. Nat. Methods 3, 99-108.

Oehring, H., Riemann, I., Fischer, P., Halbhuber, K. J., and König, K. (2000). Ultrastructure and reproduction behaviour of single $\mathrm{CHO}-\mathrm{K} 1$ cells exposed to near infrared femtosecond laser pulses. Scanning 22, 263-270.

Sacconi, L., O'Connor, R. P., Jasaitis, A., Masi, A., Buffelli, M., and Pavone, F. S. (2007). In vivo multi-photon nanosurgery on cortical neurons. J. Biomed. Opt. 12, 050502. doi:10.1117/1.2798723.

Sacconi, L., Tolić-Nørrelykke, I. M., Antolini, R., and Pavone, F. S. (2005). Combined intracellular three-dimensional imaging and selective nanosurgery by a nonlinear microscope. J. Biomed. Opt. 10, 014002-014005.

Schaffer, C. B., Friedman, B., Nishimura, N., Schroeder, L. F., Tsai, P. S., Ebner, F. F., Lyden, P. D., and Kleinfeld, D. (2006). Two-photon imaging of cortical surface microvessels reveals a robust redistribution in blood flow after vascular occlusion. PLoS Biol. 4, e22. doi:10.1371/journal.pbio.0040022.

Shen, N., Datta, D., Schaffer, C. B., LeDuc, P., Ingber, D. E., and Mazur, E. (2005). Ablation of cytoskeletal filaments and mitochondria in cells using a femtosecond laser nanoscissor. Mech. Chem. Biosyst. 2, 17-26.

Shimada, T., Watanabe, W., Matsunaga, S., Higashi, T., Ishii, H., Fukui, K., Isobe, K., and Itoh, K. (2005). Intracellular disruption of mitochondria in a living HeLa cell with a 76-MHz femtosecond laser oscillator. Opt. Express 13, 9869-9880.
Smith, N. I., Fujita, K., Kaneko, T., Katoh, K., Nakamura, O., Kawata, S., and Takamatsu, T. (2001). Generation of calcium waves in living cells by pulsed-laser-induced photodisruption. Appl. Phys. Lett. 79, 1208-1210.

Supatto, W., Débarre, D., Moulia, B. Brouzés, E., Martin, J., Farge, E., and Beaurepaire, E. (2005). In vivo modulation of morphogenetic movements in Drosophila embryos with femtosecond laser pulses. Proc. Natl. Acad. Sci. U.S.A. 102, 1047-1052.

Supatto, W., Fraser, S. E., and Vermot, J. (2008). An all-optical approach for probing microscopic flows in living embryos. Biophys. J. 95, 4.

Svoboda, K., and Yasuda, R. (2006). Principles of two-photon excitation microscopy and its applications to neuroscience. Neuron 50, 823-839.

Tirlapur, U. K., and König, K. (2002). Targeted transfection by femtosecond laser. Nature 418, 290-291.

Tolić-Nørrelykke, I. M., Sacconi, L., Thon, G., and Pavone, F.S. (2004). Positioning and elongation of the fission yeast spindle by microtubule-based pushing. Curr. Biol. 14, 1181-1186.

Trachtenberg, J. T., Chen, B. E., Knott, G. W., Feng, G., Sanes, J. R., Welker, E., and Svoboda, K. (2002). Long-term in vivo imaging of experience-dependent synaptic plasticity in adult cortex. Nature 420, 788-794.

Tsai, P. S., Blinder, P., Migliori, B. J., Neev, J., Jin, Y., Squier, J. A., and Kleinfeld, D. (2009). Plasma-mediated ablation: An optical tool for submicrometer surgery on neuronal and vascular systems. Curr. Opin. Biotechnol. 20, $1-10$.

Vogel, A., Noack, J., Hüttman, G., and Paltauf, G. (2005). Mechanisms of femtosecond laser nanosurgery of cells and tissues. Appl. Phys. B 81, 1015-1047.

Vogel, A., and Venugopalan, V. (2003). Mechanisms of pulsed laser ablation of biological tissues. Chem. Rev. 103, 577-644.

Waller, A. (1850). Experiments on the section of the glossopharyngeal and hypoglossal nerves of the frog, and observations of the alterations produced thereby in the structure of their primitive fibres. Philos. Trans. R. Soc. Lond., B, Biol. Sci. 140, 423-429.

Watanabe, W., and Arakawa, N. (2004). Femtosecond laser disruption of subcellular organelles in a living cell. Opt. Express 12, 4203-4213.
Yanik, M. F., Cinar, H., Cinar, H. N., Chisholm, A. D., Jin, Y., and Ben-Yakar, A. (2004). Neurosurgery: functional regeneration after laser axotomy. Nature 432, 822.

Zeira, E., Manevitch,A., Khatchatouriants, A., Pappo, O., Hyam, E., DarashYahana, M., Tavor, E., Honigman, A., Lewis, A., and Galun, E. (2003). Femtosecond infrared laser an efficient and safe in vivo gene delivery system for prolonged expression. Mol. Ther. 8, 342-350.

Zhang, S., Boyd, J., Delaney, K., and Murphy, T.H. (2005). Rapid reversible changes in dendritic spine structure in vivo gated by the degree of ischemia. J. Neurosci. 25, 5333-5338.

Zhang, S., and Murphy, T. H. (2007). Imaging the impact of cortical microcirculation on synaptic structure and sensory-evoked hemodynamic responses in vivo. PLoS Biol. 5, e119. doi: 10.1371/journal. pbio.0050119.

Zimmerman, U. P., and Schlaepfer, W. W. (1984). Multiple forms of Ca-activated protease from rat brain and muscle. J. Biol. Chem. 259, 3210-3218.

Zipfel, W. R., Williams, R. M., and Webb, W.W. (2003). Nonlinear magic: multiphoton microscopy in the biosciences. Nat. Biotechnol. 21, 1369-1377.

Zuo, Y., Lin, A., Chang, P., and Gan, W. B. (2005). Development of long-term dendritic spine stability in diverse regions of cerebral cortex. Neuron 46, 181-189.

Conflict of Interest Statement: The authors declare that the research was conducted in the absence of any commercial or financial relationships that could be construed as a potential conflict of interest.

Received: 26 February 2010; paperpending published: 30 April 2010; accepted: 05 July 2010; published online: 30 July 2010.

Citation: Allegra Mascaro AL, Sacconi L and Pavone FS (2010) Multi-photon nanosurgery in live brain. Front. Neuroenerg. 2:21. doi: 10.3389/fnene.2010.00021 Copyright (C) 2010Allegra Mascaro, Sacconi and Pavone. This is an open-access article subject to an exclusive license agreement between the authors and the Frontiers Research Foundation, which permits unrestricted use, distribution, and reproduction in any medium, provided the original authors and source are credited. 